ISSN 1991-8631

Original Paper

http://indexmedicus.afro.who.int

\title{
Effect of antioxidants supplementations in salt-induced dyslipidaemia in albino rats
}

\author{
Lawal Suleman BILBIS *, Yusuf SAIDU, Kabiru AHMAD, Atteeque Umar BABA and \\ Aishat Mohmmed SHUAIB \\ Department of Biochemistry, Usmanu Danfodiyo University Sokoto, PMB 2346 Sokoto, Nigeria. \\ *Corresponding author, E-mail: lsbilbis@yahoo.co.uk; lsbilbis@udusok.edu.ng; Tel +2348034227001
}

\begin{abstract}
Cardiovascular disease (CVD) is associated with many risk factors including oxidative stress and dyslipidaemia. The current work evaluated the effects of antioxidants supplementation on salt-induced dyslipidaemia in albino rats. Rats were divided into 10 groups of 7 rats each. Groups 2-10 were fed 8\% salt diets for 5 weeks while group 1 served as control and were fed normal rat feed. Water was provided to all the groups ad libitum. The animals in groups 3-10 were then supplemented with vitamin A; vitamin C; vitamin E; $\mathrm{Cu}$; $\mathrm{Mn}$; $\mathrm{Zn}$; vitamins $\mathrm{A}, \mathrm{C}$ and $\mathrm{E}$ combined and $\mathrm{Cu}, \mathrm{Mn}$ and $\mathrm{Zn}$ combined respectively for additional 4 weeks simultaneously with salt loading. Group 2 was not supplemented and served as the negative control. Serum levels of total cholesterol, triacylglyceride, low density lipoprotein (LDL) cholesterol, very low density lipoprotein (VLDL) cholesterol, high density lipoprotein (HDL) cholesterol and glucose were estimated. The results indicated that the vitamins reduced significantly serum lipid profiles and the atherogenic index by up to $80 \%$. The serum glucose levels of the rats supplemented with antioxidant vitamins and minerals were also significantly $(\mathrm{P}<0.05)$ lowered compared with the negative control group. These results suggest that the reduction of serum lipid profile and glucose level may be due to regulation of cholesterol and lipoprotein metabolism and increased insulin sensitivity as a result of the supplementations. It may thus suggest that the antioxidants may provide protection against CVDs and metabolic syndrome in salt-induced dyslipidaemia in rats.
\end{abstract}

(C) 2012 International Formulae Group. All rights reserved.

Keywords: Metabolic Syndrome, Cardiovascular disease, Atherogenic index, Micronutrients, Reactive Oxygen Species.

\section{INTRODUCTION}

Accumulating evidence indicates that oxidative stress through over production of reactive oxygen (ROS) and nitrogen species (RNS) or antioxidants deficiency plays a major role in the initiation and progression of cardiovascular dysfunction associated with diseases and conditions such dyslipidaemia, development of insulin resistance, $\beta$-cell dysfunction, impaired glucose tolerance and eventual development of type 2 diabetes, ischemic heart disease, chronic heart failure and hypertension (Grossman, 2008; Rodrigo, 2007). ROS are generated in aerobic organisms during physiological or physiopathological oxidative metabolism of mitochondria. ROS may react with a variety of biomolecules, including lipids, 
carbohydrates, proteins, nucleic acids, and macromolecules of connective tissue, thereby interfering with cell functions. Under normal physiological conditions, there is a critical balance in the generation of oxygen free radicals and antioxidant defense systems. Impairment in the oxidant/antioxidant equilibrium provokes a situation of oxidative stress and generally results from hyper production of ROS. Oxidative stress is known to be a component of molecular and cellular tissue damage mechanisms in a wide spectrum of human diseases (Valko et al., 2007).

Previous studies have demonstrated that elevated dietary salt intake leads to an impaired relaxation of blood vessels to endothelium-dependent relaxations induced by a variety of vasodilator agents (Bragulat et al., 2001; Campese, 1994; Boegehold, 1993; Guzik, 2002; Lenda et al., 2000). A possible contributor to impaired vascular relaxation to dilator stimuli in animals on a high-salt diet is an impaired function of the endothelium. An increase in blood pressure (BP) in response to dietary sodium (salt sensitivity) is a welldocumented phenomenon in humans and is considered to be an important factor in the pathogenesis of hypertension ((Bragulat et al., 2001). In animal models of salt-sensitive hypertension, the increase in BP after salt loading is characterized by reduced nitric oxide production Campese et al., 1996; Fujiwara et al., 2000). Similarly, in humans with salt-sensitive hypertension, salt loading was associated with decreased plasma and urinary levels of nitric oxide metabolites. A variety of evidence suggests that reactive oxygen species contribute to impaired endothelial function in several forms of hypertension and that there is increased oxidative stress in the microvessels of spontaneously hypertensive rats and Dahl saltsensitive hypertensive rats (Swei et al., 1997; Manning et al., 2003). A recent report by Lenda et al. (2000) has suggested that reactive oxygen species can also contribute to reduced endothelium-dependent dilation in normotensive rats on a high-salt diet.
Dyslipidaemia is defined as an abnormal lipids status. Common abnormalities include elevated levels of total cholesterol, low density lipoprotein cholesterol (LDL c), $\mathrm{Lp}$ (a) and triglyceride; low levels of high density cholesterol (HDL c) and a preponderance of small dense LDL particles. These abnormalities can be found alone or in combination. Dyslipidemia, very common in patients with type 2 diabetes is one of the risk factors of cardiovascular disease. Many studies have indicated that in diabetes it is not glycemia but hypertension, serum lipids, and body mass index that are independently associated with the risk of developing diabetic neuropathy (Brismar et al., 2007). Increased levels of plasma lipids, either causative of or subsequent to the development of diabetes, act in concert with both oxidative stress and glucose to produce peripheral sensory neuron and microvessel injury leading to diabetic neuropathy (Jha et al., 1995). Diabetic dyslipidemia is generally characterized by increased plasma triglyceride (TG) and decreased high-density lipoprotein (HDL)cholesterol (HDL-C) concentrations, a preponderance of small dense low-density lipoprotein (LDL), and an increased apo lipoprotein B concentration. Although the major focus on the connection between lipids and CHD is on LDL-cholesterol (LDL-C), the Adult Treatment Panel III has recognized the important roles of HDL-C and TGs, calling this combination an atherogenic dyslipidemia (Cleeman, 2001).

To protect the cells and organ systems of the body against reactive oxygen and nitrogen species, organisms have evolved a highly sophisticated and complex antioxidants protection system both exogenous and endogenous in origin, that functions interactively and synergistically to neutralize excess free radicals. Antioxidants are also employed to alleviate vasomotor disturbances in hypercholesterolemia and to slow the progression of atherosclerosis (Carlene et al., 2004). For this reason, maintenance and enhancement of antioxidant capacity is important. However, single antioxidants are 
not generally effective in patient trials, probably because of the long term requirements for drug efficacy and the counterproductive effects of long term use of high dose single antioxidants (Jha et al., 1995).

In this study the effects of supplementing with some antioxidants vitamins and minerals in reversing saltinduced dyslipidaemia and other metabolic syndrome-like conditions are investigated.

\section{MATERIALS AND METHODS Experimental animals}

Fifty five (50) adult male Sprague Dawley rats (gross body weight 150-200 g) procured from the Animal house of Biological Sciences Department, Usmanu Danfodiyo University Sokoto were acclimatized under controlled conditions of humidity with regular light/dark cycle and had free access to food and water for one week before use. Following acclimatization, the animals were randomly allocated to 10 groups $(n=7)$ and labeled as group 1 to 10 . Salt loading was used to induce dyslipidaemia in Groups 2 to 10 by supplementing with $8 \%$ common salt in their diet for five (5) weeks. Group 1 was fed nonsalt supplemented diet for the same period.

\section{Antioxidants supplementation}

Group 1 served as positive control and was fed standard rat chow while group 2 was also feed standard rat chow and served as negative control. Group 3 was supplemented with vitamin A (7.16 mg/kg body weight), Group 4 vitamin C (16.67 mg/kg body weight), Group 5 vitamin $\mathrm{E}(13.17 \mathrm{mg} / \mathrm{kg}$ body weight), Group 6 copper $(4.5 \mathrm{mg} / \mathrm{kg}$ body weight), Group 7 manganese $(2.3 \mathrm{mg} / \mathrm{kg}$ body weight), Group 8 zinc $(2.0 \mathrm{mg} / \mathrm{kg}$ body weight), Group 9 all the vitamins combined and Group 10 the minerals combined, for a period of four (4) weeks.

Body weight and pulse rates of the animals were monitored weekly. At the end of the experiment the animals were anaesthetized in chloroform vapor and blood sample collected by cardiac puncture for Biochemical analysis. Fasting blood sugar was estimated by glucose oxidase method (Cheesbrough, 1992). Serum total cholesterol (TC) (Allain et al., 1974), high density lipoprotein (HDL) (Doumas, 1975) and Triacylglycerol (TAG) (Cheesbrough, 1992) were also determined by enzymatic methods, while Low density lipoprotein (LDL) and very low density lipoprotein (VLDL) cholesterol were calculated using Friedwald formula (Friedewald et al., 1972). Atherogenic index LDL/HDL and Atherogenic Index of the plasma (AIP) were computed (Meng et al., 2004).

\section{Statistical analysis}

All data were expressed as mean \pm standard deviation. Statistical analysis was carried out by one-way ANOVA. Post hoc test for multiple comparison using LSD were utilized to identify differences in means. Statistical significance was considered at $\mathrm{P}<$ 0.05 .

\section{RESULTS}

The results of the plasma lipid profiles, AI and AIP are shown in Table 1. All lipid fractions of group 1 demonstrated normal baseline level. When groups 2 to 10 were supplemented with $8 \%$ salt for 5 weeks, dyslipidaemia occurred as indicated by marked increase of TCHOL, LDL and TG levels $(\mathrm{p}<0.05)$ in comparison with the levels in group 1. The increase of TCHOL, LDL and TG, FBS and AI levels of groups 2 to 10 was found to be about 53, 55, 79, 34 and $82 \%$ higher than that of group 1, respectively. It could be seen that $8 \%$ salt loading resulted in dyslipidaemia and metabolic syndrome-like conditions that is increased triglyceride, LDL cholesterol, decrease HDL cholesterol levels, insulin resistance and hyperglycaemia. The implication is that salt loading induced metabolic syndrome that was reversed by supplementation with the antioxidants. It could be possible that feeding the animals with $8 \%$ salt exerts oxidative stress but treating the animals with antioxidants resulted in the correction of the conditions even in the presence of the challenging agent ( $8 \%$ salt). 
Table 1: Effect of supplementation on lipid profile of salt induced-hypertensive rats.

\begin{tabular}{|c|c|c|c|c|c|c|c|c|}
\hline Group & $\begin{array}{c}\text { Total Cholesterol } \\
(\mathbf{m M})\end{array}$ & $\begin{array}{l}\text { TAG } \\
(\mathbf{m M})\end{array}$ & $\begin{array}{c}\text { LDL-C } \\
(\mathrm{mM})\end{array}$ & $\begin{array}{c}\text { VLDL-C } \\
(\mathbf{m M})\end{array}$ & $\begin{array}{l}\text { HDL-C } \\
(\mathrm{mM})\end{array}$ & $\begin{array}{l}\text { Fasting blood } \\
\text { Sugar }(\mathbf{m M})\end{array}$ & $\begin{array}{l}\text { Atherogen } \\
\text { ic Index } \\
\text { (AI) }\end{array}$ & $\begin{array}{c}\text { Atherogenic } \\
\text { Index of Plasma } \\
\text { (AIP) }\end{array}$ \\
\hline $\mathrm{NU}$ & $1.54 \pm 0.27$ & $0.82 \pm 0.06$ & $0.51 \pm 0.29$ & $0.16 \pm 0.01$ & $0.86 \pm 0.33$ & $3.59 \pm 0.91$ & 0.59 & -0.02 \\
\hline $\mathrm{HU}$ & $3.26 \pm 0.18^{\mathrm{a}}$ & $1.81 \pm 0.07^{\mathrm{a}}$ & $2.39 \pm 0.19^{\mathrm{a}}$ & $0.16 \pm 0.02$ & $0.71 \pm 0.02$ & $5.40 \pm 0.35^{\mathrm{a}}$ & $3.37^{\mathrm{a}}$ & 0.41 \\
\hline HTVA & $0.95 \pm 0.41^{\mathrm{b}}$ & $0.83 \pm 0.29^{b}$ & $0.17 \pm 0.10^{\mathrm{ab}}$ & $0.17 \pm 0.06$ & $0.62 \pm 0.26$ & $3.42 \pm 0.32^{\mathrm{b}}$ & $0.27^{\mathrm{b}}$ & 0.13 \\
\hline HTVC & $0.73 \pm 0.58^{b}$ & $0.94 \pm 0.15^{\mathrm{b}}$ & $0.14 \pm 0.10^{\mathrm{ab}}$ & $0.19 \pm 0.03$ & $0.48 \pm 0.30$ & $4.61 \pm 0.33$ & $0.29^{\mathrm{b}}$ & 0.29 \\
\hline HTVE & $1.26 \pm 0.33^{b}$ & $0.99 \pm 0.04^{\mathrm{b}}$ & $0.28 \pm 0.16^{\mathrm{b}}$ & $0.19 \pm 0.01$ & $0.79 \pm 0.17$ & $4.20 \pm 0.28$ & $0.35^{\mathrm{b}}$ & 0.01 \\
\hline $\mathrm{HTCu}$ & $1.97 \pm 0.32^{\mathrm{b}}$ & $1.29 \pm 0.91$ & $0.84 \pm 0.43^{b}$ & $0.26 \pm 0.18$ & $0.87 \pm 0.52$ & $3.72 \pm 0.82^{\mathrm{b}}$ & $0.97^{\mathrm{b}}$ & 0.17 \\
\hline HTMn & $1.17 \pm 0.57^{\mathrm{b}}$ & $0.81 \pm 0.20^{\mathrm{b}}$ & $0.29 \pm 0.18^{b}$ & $0.16 \pm 0.04$ & $0.75 \pm 0.35$ & $5.01 \pm 1.0^{\mathrm{a}}$ & $0.39^{\mathrm{b}}$ & 0.03 \\
\hline HTZn & $2.81 \pm 0.79^{\mathrm{a}}$ & $0.76 \pm 0.05^{\mathrm{b}}$ & $0.75 \pm 0.56^{\mathrm{b}}$ & $0.16 \pm 0.01$ & $1.94 \pm 0.4^{\mathrm{ab}}$ & $5.85 \pm 1.19^{\mathrm{a}}$ & $0.39^{\mathrm{b}}$ & -0.41 \\
\hline HTAVC & $2.52 \pm 0.58^{\mathrm{a}}$ & $0.76 \pm 0.17^{\mathrm{b}}$ & $1.14 \pm 0.83^{\mathrm{ab}}$ & $0.15 \pm 0.03$ & $1.32 \pm 0.85$ & $4.0 \pm 0.31$ & $0.86^{\mathrm{b}}$ & -0.24 \\
\hline HTAMC & $2.03 \pm 0.97^{\mathrm{b}}$ & $0.81 \pm 0.26^{\mathrm{b}}$ & $0.81 \pm 0.04^{b}$ & $0.15 \pm 0.03$ & $1.06 \pm 0.78$ & $5.79 \pm 0.30^{\mathrm{a}}$ & $0.76^{\mathrm{b}}$ & -0.01 \\
\hline
\end{tabular}

a: values bearing "a" differ significantly from NU; while those bearing "b" differ significantly from HU using LSD; p<0.05; NU: Normal untreated; HU: Hypertensive untreated; HTVA: Hypertensive treated with Vitamin A; HTVC: Hypertensive treated with Vitamin C; HTVE: Hypertensive treated with Vitamin E; HTCu: Hypertensive treated with Copper; HTMn: Hypertensive treated with Manganese; HTZn: Hypertensive treated with Zinc; HTAVC: Hypertensive treated with all Vitamins Combined; HTAMC: Hypertensive treated with all Minerals combined; TAG: Triacylglycerol; HDL-C : High density lipoprotein cholesterol; LDL-C: low density lipoprotein cholesterol; VLDL-C: very low density lipoprotein cholesterol; Aix: Atherogenic index. 
Of the vitamins, vitamin $\mathrm{A}$ is the most effective in correcting the salt-induced dyslipidaemia by reducing TC by up $70 \%$ and LDL by $93 \%$ (Table 1) but non-significantly ( $\mathrm{p}<0.05)$ reduces HDL. It also reduced atherogenic index by $92 \%$ and atherogenic index of plasma by $68 \%$. Vitamin C significantly reduced total cholesterol, triacylglyceride, LDL, AI, AIP and fasting blood glucose by 78, 48, 94, 91, 29 and $15 \%$ respectively. Only Vitamin $\mathrm{E}$ had any significant effect on HDL. The minerals seemed to have much effect on TAG and HDL, where $\mathrm{Mn}$ and $\mathrm{Zn}$ normalized TAG with $\mathrm{Zn}$ increasing HDL levels three fold and normalized AIP.

The results indicated that the antioxidants reduced significantly serum lipid profiles and the atherogenic index by up to $90 \%$. The serum glucose levels of the rats supplemented with antioxidant vitamins and minerals were also significantly $(\mathrm{P}<0.05)$ lowered compared with the negative control group. These results suggest that the reduction of serum lipid profile and glucose level may be due to regulation of cholesterol and lipoprotein metabolism and increased insulin sensitivity as a result of the supplementations. It may thus suggest that the antioxidants may provide protection against CVDs and metabolic syndrome if intervention starts earlier before the onset of irreversible stage.

\section{DISCUSSION}

Reactive oxygen species (ROS) generated in vivo is responsible for the oxidative damage of lipids, proteins, DNA and small molecules. Recent evidence also indicates that oxidative stress is the main mechanism responsible for cardiovascular diseases while hypercholesterolemia under oxidative stress is the predisposing factor for the progression of atherosclerosis and abnormal lipid metabolism. On the other hand, antioxidants might be able to prevent the impairment originated by excessive oxidative stress. Since endogenous antioxidants may not be sufficient to prevent damage, diet derived antioxidants could be important for maintaining health and preventing free radical-mediated diseases (Zulkhairi et al., 2010). In this study $8 \%$ salt loading to the animals generated dyslipidaemia and other metabolic syndrome risk factors. Studies in both animals and human have demonstrated that prolonged high salt correlates with impaired endothelium function and pathogenesis of hypertension (Bragulat et al., 2001). High salt is reported to reduce the level nitric oxide (NO) by decreasing the NO synthase thus promoting hypertension and oxidative stress (Anees et al., 2007). From the results of this study it could be suggested that the antioxidants supplementation reversed the salt-induced dyslipidaemia and all other metabolic risk factors even in the present of the challenging agent, the salt.

Important application of plasma lipid markers that is relevant to CVD risk is the association between the LDL to HDL ratio known as atherogenic index (AI). Our results showed that antioxidants vitamins and minerals had a strong hypocholesterolemic effects in plasma of rats with a reduction of plasma TCHOL, TAG, LDL and AI with only vitamin $\mathrm{E}, \mathrm{Cu}, \mathrm{Mn}, \mathrm{Zn}$ and combination of the vitamins having significant effect on HDL levels (Table 1). Furthermore, the atherogenic index is markedly decreased due to a significant decrease of LDL levels. The observed increase of HDL is one of the most important criteria of an antihypercholesterolemic agent. Moreover, numerous studies have demonstrated that high levels of HDL are associated with a lower incidence of cardiovascular diseases (CVD) (Catherine et al., 2004). Studies have demonstrated that lipid lowering agents from several pharmacological classes reduce the susceptibility of LDL to oxidation, and it has been suggested that this is not entirely caused by LDL cholesterol reduction but by antioxidants activity (Hoffman et al., 1992). The increase in HDL levels observed in our study might be due to the stimulation of preHDL and reverse cholesterol transport as shown by previous findings (Gupta et al., 1993). The observed hypocholesterolaemic effect of the vitamin $\mathrm{C}$ and the antioxidant minerals in our study could be the basis for suggesting therapeutic potential of ascorbate in reducing atherosclerotic risk via inhibition of $\mathrm{H}_{2} \mathrm{O}_{2}$-induced oxidative stress in patients 
with Uremia or dyslipidaemia (Chiang et al., 2004).

In this study zinc supplementation was found to have antiatherogenic properties by reducing atherogenic index by $88 \%$ and normalizing atherogenic index of plasma, other researchers have demonstrated the antiatherogenic properties of zinc that might be by preventing metabolic physiologic derangement of the vascular endothelium (Hennig et al., 1996). Because of its antioxidant and membrane-stabilizing properties, zinc appears to be crucial for the protection against cell-destabilizing agents. The antiatherogenic role of zinc appears to be in its ability to inhibit oxidative stress responsive factors (Hennig et al., 1996).

Atherogenic Index of Plasma (AIP), defined as log (TG/HDL-C), has recently been proposed as a marker of plasma atherogenicity because it is increased in people at higher risk for coronary heart disease and is inversely correlated with LDL particle size. AIP correlates with the size of pro- and anti atherogenic lipoprotein particles. Clinical studies have shown that AIP predicts cardiovascular risk. AIP is an easily available cardiovascular risk marker and a useful measure of response to treatment (pharmacological intervention) (Meng et al., 2004). In this study Zinc was found to normalize AIP followed by combined vitamins and combined minerals.

\section{ACKNOWLEDGEMENTS}

This work is carried out with the financial assistance of the World Bank assisted program of Federal Ministry of Education of Nigeria through the Science and Technical Education Post Basic (STEP-B) Program.

\section{REFERENCES}

Allain CC, Poon LS, Chan CSG, Richmond W, Fu PC. 1974. Enzymatic determination of total serum cholesterol. Clinical Chemistry, 20: 470.

Anees A B, Abdulbari M, Fatima RF, Mustafa L. 2007. Mechanisms of oxidative stress-induced increase in salt sensitivity and development of hypertension in
Sprague-Dawley rats. Hypertension, 49: 664-666.

Boegehold MA. 1993. Effect of dietary salt on arteriolar nitric oxide in striated muscle of normotensive rats. Am. J. Physiol., 264: H1810-H1816.

Bragulat E, de la Sierra A, Antonio MT, Coca A. 2001. Endothelial dysfunction in saltsensitive essential hypertension. Hypertension, 37: 444-448.

Brismar T, Maurex L, Cooray G. 2007. Predictors of cognitive impairment intype1diabetes. Psychoneuroendocrinology, 32(8-10): 1041-1051.

Campese VM, Tawadrous M, Bigazzi R, Bianchi S, Mann AS, Oparil S, Raij L. 1996. Salt intake and plasma atrial natriuretic peptide and nitric oxide in hypertension. Hypertension, 28: 335340.

Campese VM. 1994. Salt sensitivity in hypertension: renal and cardiovascular implications. Hypertension, 23: 531-550.

Carlene AH, William H, Miller S, AL-benna M, Julia B, Russell DD, Martin WM, Anna FD. 2004. Strategies to reduce oxidative stress in cardiovascular disease. Clinical Science, 106: 219-234.

Catherine N, Nicolas C, Elyett G, Lydia J, Edmond R, Andrzej M, Pierre A, Christian R. 2004. Health effect of vegetable-based diet: lettuce consumption improves cholesterol metabolism and antioxidant status in the rat. J. Clin. Nutr., 23: 605-614.

Ceconi C, Boraso A, Cargnoni A, Ferrari R. 2003. Oxidative stress in cardiovascular disease: myth or fact? Arch. Biochem. Biophys. 420(2): 217-221.

Cheesbrough M. 1992. Medical Laboratory Manual for Tropical Countries (vol 1, $2^{\text {nd }}$ edn). ELBS: Cambridge; 527-545.

Chiang T, Wei-Tien C, Heui-Wen C, TzungDau W, Shaw Y, Tzay-Jinn C, Yen-Lin C, Yuan-Teh L, Su-Ming H. 2004. Ascorbate Supplement Reduces Oxidative Stress in Dyslipidemic Patients Undergoing Apheresis. Arteriosclerosis, Thrombosis, and Vascular Biology, 24: 1111-1117. 
Cleeman J. 2001. Executive summary of the Third Report of the National Cholesterol Education Program (NCEP) Expert Panel on Detection, Evaluation, and Treatment of High Blood Cholesterol in Adults (Adult Treatment Panel III). JAMA, 285: 2486-2497.

Doumas BT. 1975. Standards for total serum protein assays-a collab- orative study. Clinical Chemistry, 21: 1159-1166.

Friedewald WT, Levy RI, Fredrickson DS. 1972. Estimation of the concentration of low-density lipoprotein cholesterol in plasma, without the use of the preparative ultracentrifuge. Clinical Chemistry, 18(6): 499-502.

Fujiwara N, Osanai T, Kamada T, Katoh T, Takahashi K, Okumura K. 2000. Study on the relationship between plasma nitrite and nitrate level and salt sensitivity in human hypertension: modulation of nitric oxide synthesis by salt intake. Circulation, 101: 856-861.

Grossman E, 2008. Does Increased Oxidative Stress Cause Hypertension? Diabetes Care, 31(2): S185-S189.

Gupta AK, Ross EA, Myers JN, Kashyap ML. 1993. Increased reverse cholesterol transport in athelets. Metabolism, 42: 684-690.

Guzik TJ, West NE, Pillai R, Taggart DP, Channon KM. 2002. Nitric oxide modulates superoxide release and peroxynitrite formation in human blood vessels. Hypertension, 39: 1088-1094.

Hennig B, Toborek M, Mcclain CJ. 1996. Antiatherogenic properties of zinc: implication in endothelial metabolism. Nutrition, 12(10): 711-717.

Hoffman R, Brook GJ, Aviram M. 1992. Hypolipidemic drugs reduce lipoprotein susceptibility to undergo lipid peroxidation: in vitro and in vivo studies. Atherosclerosis, 93: 105-113.

Jha P, Flather M, Lonn E, Farkouh M, Yusuf S. 1995. The antioxidant vitamins and cardiovascular disease. A critical review of epidemiologic and clinical trial data. Ann. Intern. Med., 123(11): 860-872.

Lenda DM, Sauls BA, Boegehold MA. 2000. Reactive oxygen species may contribute to reduced endothelium-dependent dilation in rats fed high salt. Am. J. Physiol., 279: H7-H14.

Manning RD Jr, Meng S, Tian N. 2003. Renal and vascular oxidative stress and saltsensitivity of arterial pressure. Acta. Physiol. Scand., 179: 243-250.

Meng HT, Don J, Bradly NG. 2004. Pioglitazone Reduces Atherogenic Index of Plasma in Patients with Type 2 Diabetes. Clinical Chemistry, 50: 11841188.

Rodrigo R, Prat H, Passalacqua W, Araya J, Guichard C, Bächler JP. 2007. Relationship between Oxidative Stress and Essential Hypertension. Hypertens. Res., 30: 1159-1167.

Swei A, Lacy F, DeLano FA, SchmidSchonbein GW. 1997. Oxidative stress in the Dahl hypertensive rat. Hypertension, 30: 1628-1633.

Valko M, Leibfritz D, Moncol J, Cronin MT, Mazur M, Telser J.2007. Free radicals and antioxidants in normal physiological functions and human disease. Int. J. Biochem. Cell Biol., 39: 44-84.

Zulkhairi AF, Khairunnuur MRN, Hafipah A, Azrina MA, Rasadah KAK, Kamilah MS, Zamree A, Shahidan MA. 2010. J. Med. Plant Res., 4(1): 049-057. 